\title{
Überlegungen zur (adäquaten) lexikografischen Beschreibung des (,politisch korrekten') Gebrauchs von Wörtern wie Ausländer, Migrant, Flüchtling, Asylbewerber
}

\begin{abstract}
Bezeichnungen für Personen, die sich nicht in ihrem Heimatland aufhalten (z.B. Migrant, Ausländer, Flüchtling) werden in der Sprachgemeinschaft häufig wertend und kontrovers verwendet. In dem Beitrag wird gezeigt, dass die allgemeinsprachige Lexikografie diesen Aspekt bislang nicht angemessen berücksichtigt - weder in der korpusgestützten, methodischen Erfassung und Analyse von Sprachdaten noch in der beschreibenden Darstellung. Am Beispiel von elexiko werden Ansätze vorgestellt, die das Potenzial besitzen, dieses Desiderat einzulösen.

Terms designating people who do not live in their home country (e.g. migrants, foreigners, refugees) are often used controversially along with judgmental connotations by a speech community. In this paper, I will demonstrate that general monolingual lexicography has so far not considered this aspect appropriately neither in corpus-assisted, methodological analysis of language data nor in descriptive documentation. With the help of some entries taken from elexiko, approaches are being discussed which might have the potential to achieve this desideratum.
\end{abstract}

\section{Annäherung}

Das Spektrum an Bezeichnungen für Personen, die sich nicht in ihrem Heimatland aufhalten, ist sehr groß: Asylant, Asylbewerber, Asylbewerberin, Asylsuchender, Asylsuchende, Ausländer, Ausländerin, Aussiedler, Auswanderer, Bootsflüchtling, Bürgerkriegsflüchtling, Emigrant, Einwanderer, Flüchtling, Fremdarbeiter, Gastarbeiter, Immigrant, Immigrantin, Kriegsflüchtling, Neuankömmling, Neubürger, Saisonarbeiter, Spätaussiedler, Übersiedler, Urlauber, Vertriebener, Wanderarbeiter, Wirtschaftsflüchtling, Zuwanderer usw. ${ }^{1}$

Liest man diese Aufzählung von Wörtern (laut), so werden sie einem wahrscheinlich mit unterschiedlichen ,Gefühlswelten' über die Lippen gehen. Auch wenn diese Bezeichnungen im Bedeutungskern Überschneidungen aufweisen, verdeutlicht z.B. die Gegenüberstellung von Neubürger oder gar Urlauber und Asylant wohl am eindringlichsten, dass in diesem Feld eine möglichst genaue Beschreibung der Bedeutung im Sinne der präzisen Sprachverwendung notwendig ist. Das Spektrum der Wörter ist breit gefächert, von (relativ) neutral verwendbar bis stark abwertend konnotiert. Beispielsweise im Rahmen der (journalistischen) Textproduktion mag es zu Unsicherheiten und Problemen bei der Wahl der ,politisch korrekten“ Personenbezeichnung kommen. Das zeigen auch zahlreiche Sprachanfragen, die im Institut für Deutsche Sprache eingehen. Doch auf welche Hilfsmittel könnten Ratsuchende in Situationen sprachlicher Unsicherheiten überhaupt zurückgreifen?

Die Zusammenstellung wurde mit Hilfe des Analyseverfahrens ,Modelling Semantic Proximity: Similar Collocation Profiles‘ auf der Basis der Kollokationsdatenbank CCDB ermittelt, einem Verfahren, das Ausdrücke mit ähnlichen kontextuellen Verwendungsaspekten ermittelt. Suchwörter waren: Ausländer und Gastarbeiter (vgl. Belica 1995 und 2001ff.). 
In der wissenschaftlichen Literatur findet man zahlreiche einschlägige Untersuchungen, die sich mit der sprachlichen Thematisierung der Einwanderung befassen (vgl. z.B. Jung/ Niehr/Böke 1997, 2000; Niehr/Böke (Hg.) 2000; Wengeler 1995 und 2010).

Speziell für Journalisten und Journalistinnen (doch nicht nur für diese) wurde ein Leitfaden für den rassismuskritischen Sprachgebrauch (AntiDiskriminierungsBüro (ADB) Köln/ Öffentlichkeit gegen Gewalt e.V. (Hg.) 2013) erarbeitet. Im Vorwort (ebd., S. 3) heißt es:

\begin{abstract}
„Das konnte ich doch nicht wissen!“ Dies ist der häufigste Satz, den wir zu hören bekommen, wenn wir Kollegen und Kolleginnen in der Redaktion darauf aufmerksam machen, dass ihre Sprache, ihre Fotos, ihre Berichterstattung bei Themen, die Migranten betreffen, nicht angemessen sind. Aber ist es wirklich so - kann man das nicht wissen? Dass es einen Unterschied gibt zwischen ,Ausländerfeindlichkeit' und Rassismus, dass die Worte ,Farbiger' und ,Schwarzer' nicht synonym sind? Dass das Bild einer kopftuchtragenden Frau nicht die beste Wahl ist, um einen Artikel über Islamismus zu bebildern? Dass Afrika von Völkern und nicht von Stämmen bewohnt wird. Man muss es nicht wissen, aber man könnte - wenn, ja wenn man über diese Themen nachdenken würde. Vielen weißen deutschen Journalisten sind die Belange von Migranten, Schwarzen Deutschen [sic!] oder Flüchtlingen nicht wichtig genug, um sich ernsthaft damit auseinanderzusetzen, ob ihre Berichterstattung über sie adäquat ist.
\end{abstract}

Es gibt also sehr gute Gründe, sich zu informieren - und das bezieht sich nicht nur auf Journalistinnen und Journalisten! Die beiden vorgestellten Quellen eignen sich allerdings nur sehr bedingt für ,schnelles` Nachschlagen. Man fragt sich: Welche Hilfestellung könnten denn Wörterbücher in solchen und ähnlich gelagerten Fällen bieten (vgl. dazu auch Elsner-Petri in Druck)? Folgerichtig bedarf es insbesondere bei den sprachgemeinschaftlich kontrovers gebrauchten Begriffen wie Ausländer, Migrant, Flüchtling, Asylbewerber usw. besonderer Sorgfalt bei der lexikografischen Beschreibung in Wörterbüchern: Legt man den Sprachgebrauch, wie er sich beispielsweise in Korpora manifestiert, zugrunde, ist es gewiss nicht ausreichend, lediglich eine kurze Bedeutungsparaphrase (sowie ggf. unkommentierte Beispielsätze bzw. Belege) anzugeben, um die Verwendung lexikografisch umfassend und angemessen darzustellen. Insbesondere in diesen Fällen gilt es also, differenzierter vorzugehen.

\title{
2. Bestandsaufnahme
}

Die Lexikografie befindet sich seit einigen Jahren im Umbruch: Sprachrecherchen in OnlineSuchmaschinen sowie Informationsaustausch in Foren ersetzen vielfach das Nachschlagen in einem gedruckten bzw. Onlinewörterbuch. Vergegenwärtigt man sich allerdings die Qualität und Zuverlässigkeit der Suchergebnisse, so ist es doch überraschend, dass sich offenbar trotzdem eine große Zahl von Nutzer/innen damit zufrieden gibt. Nachvollziehbar scheint dies, wenn man die relativ allgemein bzw. punktuell ausgerichteten Nachschlagebedürfnisse betrachtet, etwa in der Art ,Was bedeutet vegan?‘, ,Wie schreibt man Akquise?' usw. Daneben gibt es aber auch sprachliche Unsicherheiten, die sich schwerlich in einem Satz klären lassen, etwa: ,Sagt man (noch) Gastarbeiter?', ,Was bedeutet es, wenn in einem aktuellen Text Asylant verwendet wird?' usw. Die Klärung derartiger Erkenntnisinteressen fordert den kompetenten und gewissenhaften Umgang bei der Suche in Primärquellen und der Interpretation von Suchergebnissen sowie ein gehöriges Maß an Geduld und Zeit. Daher böte es sich an, in solchen Fällen zu einem guten und zuverlässigen, d.h. in der Regel auch redaktionell aufbereiteten und nicht ausschließlich automatisiert computergenerierten Wörterbuch zu greifen. Vielleicht ist es gerade die Beschreibung bestimmter Wortschatz-Teilbereiche im Rahmen allgemeinsprachiger lexikografischer Prinzipien, z.B. also diskursrelevanter Wörter, von denen die Lexikografie trotz oder gerade aufgrund der Umbrüche in den letzten zehn Jahren in besonderem Maße profitieren könnte: Die empirische Fundierung, also die korpusgestützte lexiko- 
grafische Arbeit, garantiert eine v.a. für die Beschreibung solcher Wortschatzausschnitte wichtige Voraussetzung, nämlich die Orientierung am Sprachgebrauch. Die Verwendung von Wörtern wie Gastarbeiter oder Asylant ist an einen (engen) zeitlichen und diskursiven Rahmen gebunden, Bedeutungs- bzw. Bewertungsnuancen sind daher in gewisser Weise kontextsensitiv und auf der Zeitachse der Variabilität unterworfen - was eine ,einfache " und Bestand habende Beschreibung (z.B. im Gegensatz zu Fahrrad, schwimmen, schlank) in dieser Beziehung erschwert. In traditionellen allgemeinsprachigen Wörterbüchern findet man zahlreiche Belege für die mitunter nur wenig befriedigenden Beschreibungsversuche (s.u.). Lösungsansätze bieten hingegen spezielle sprachreflexive bzw. Diskurswörterbücher (z.B. Strauß/Haß/ Harras 1989; Stötzel/Eitz (Hg.) 2002) oder Publikationen vom Typ Diskurswörterbuch (z.B. zum ,Protestdiskurs 1967/68` vgl. Kämper 2013). Flankiert werden diese von wissenschaftlichen, quasi monographischen Veröffentlichungen, die sich beispielsweise mit den Themen „Sprache des Migrationsdiskurses‘ (Jung/Niehr/Böke 1997) oder ,Ausländer und Migranten im Spiegel der Presse“ (Jung/Niehr/Böke 2000) beschäftigen. Um diese i.d.R. eher ausführlich narrativ konzipierten Darstellungstypen geht es hier nicht, und es darf gemutmaßt werden, dass ihr Bekanntheitsgrad außerhalb wissenschaftlicher bzw. sehr interessierter Kreise eher gering ist. Sie können und soll(t)en auch deshalb sehr wohl als Anregung, Muster, als Hilfsmittel (zur Ergänzung der Korpora) in der allgemeinsprachigen, i.d.R. breitere Schichten erreichenden Lexikografie herangezogen werden.

Im Folgenden werden die Möglichkeiten der Analyse und Dokumentation diskursrelevanter Wörter mit den Mitteln und im Rahmen der modernen allgemeinsprachigen Lexikografie reflektiert. Hintergrund sind die Erfahrungen bei der Arbeit am Onlinewörterbuch elexiko, einem umfangreichen allgemeinsprachigen Nachschlagewerk zur deutschen Gegenwartssprache (www.elexiko.de). Zur Illustration dienen einige Stichproben aus dem Bereich der Bezeichnungen für Personen mit Migrationshintergrund.

Wenn Nutzer und Nutzerinnen in allgemeinen gedruckten oder Onlinewörterbüchern beispielsweise Wörter wie Flüchtling oder Asylbewerber nachschlagen, finden sie zumeist Einträge folgender Art, im „Duden Universalwörterbuch“ etwa:

(1) Flüchtling, der; -s, -e,: jmd., der aus politischen, religiösen od. ethnischen Gründen seine Heimat eilig verlassen hat od. verlassen musste u. dabei seinen Besitz zurückgelassen hat: als politischer F. anerkannt werden; das Elend der -e nach dem Krieg;

(2) Asylbewerber, der: jmd., der um Asyl ansucht

Im „Duden Bedeutungswörterbuch“ gibt es zu Asylbewerber noch einen (unkommentierten) Beispielsatz:

(3) Asyl|be|wer|ber [a'zy:lbəverbe], der; -s, -, Asyl|be|wer|be|rin [a'zy:lbəverbərın], die; -, -nen: Person, die [politisches] Asyl beantragt: der Asylbewerber lebt in einem Heim.

Auch das „PONS-Wörterbuch Deutsch als Fremdsprache (online)“ beschränkt sich auf ein absolutes Mindestmaß an sprachlicher Information, ohne auf etwaige Verwendungsbesonderheiten hinzuweisen (die insbesondere auch für Deutschlernende wichtig wären):

(4) der (die) Asyl·be·wer·ber (Asyl·be $\cdot$ wer·be $\cdot$ rin) <-s, -> jd, der den Antrag stellt, in einem Land Asyl ${ }^{1}$ zu erhalten

Flüchtling wird im „PONS-Wörterbuch Deutsch als Fremdsprache (online)“ erklärt als ,jd, der vor etwas flüchtet oder geflüchtet ist“, die zusätzlich angegebenen Fügungen „ein politischer Flüchtling“ sowie „Flüchtlinge aufnehmen/unterbringen“, vor allem aber die 
Angabe von Komposita wie -selend, -slager, -sstrom, -swelle sollten von den Nutzer/ innen wohl auch als Hinweise auf Gebrauchsbesonderheiten gedeutet werden.

Auch „Duden online“ umschreibt Asylbewerber mit der wenig hilfreichen semantischen Paraphrase ,jemand, der um Asyl (2) nachsucht“, Verwendungsbesonderheiten werden nicht eigens angeführt. Allerdings lassen einige der computergenerierten ,Typischen Verbindungen' mit Adjektiven, Substantiven und Verben Thematisierungen durchscheinen, die auch Rückschlüsse auf brisante Verwendungsweisen nahelegen - ohne diese beispielsweise in einem lexikografischen Kommentar explizit zu machen oder durch Belege zu illustrieren:

- Adjektive: afrikanisch, algerisch, anerkannt, kurdisch, minderjährig, togoisch, schwarzafrikanisch, sudanesisch.

- Verben: abschieben, anerkennen, aufnehmen, kommen, leben, niederstechen, unterbringen, verbrennen.

- Substantive: Ausländer, Aussiedler, Einwanderer, Flüchtling, Gastarbeiter, Kriegsflüchtling, Sozialhilfeempfänger, Spätaussiedler.

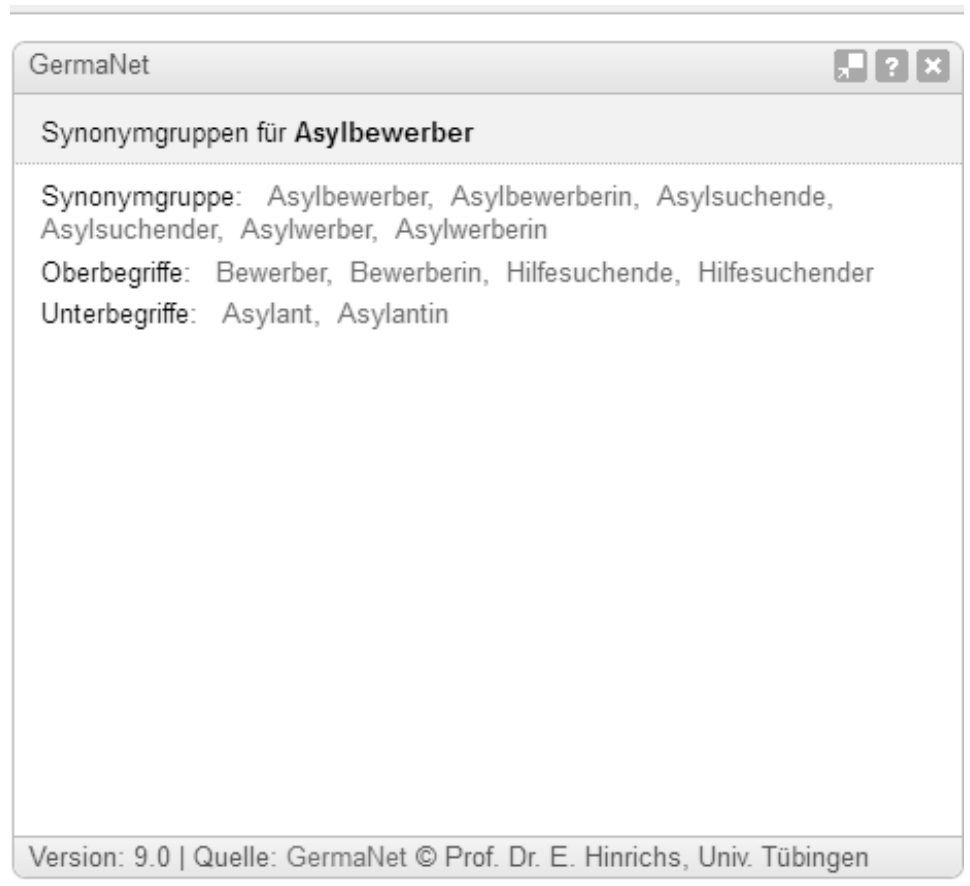

Abb. 1: Eintrag Asylbewerber, Ausschnitt (Panel) Synonymgruppe im „DWDS“

Das „Digitale Wörterbuch der Deutschen Sprache (DWDS)“ besitzt für Asylbewerber keinen eigenen Wörterbucheintrag ${ }^{2}$, dafür lassen aber die Synonymgruppen, die Attribute unter ,Wortprofil', sowie kurze Kontextausschnitte (KWICs) für (geübte) Nutzer/innen Rückschlüsse darauf zu, dass die Verwendung von Asylbewerber in einem mitunter als sensibel zu umschreibenden gesellschaftspolitischen Diskurs stattfindet (vgl. Abb. 1 bis 3). Die Daten, die aus umfangreichen Korpora ermittelt werden, führen mangels redaktioneller Rückkopplung speziell in diesem Bereich also nicht zu speziell formulierten Anga-

2 Eine Erklärung hierfür könnte sein, dass das in den 1970er Jahren in der DDR erarbeitete „Wörterbuch der deutschen Gegenwartssprache“ einen zentralen lexikografischen Baustein des „DWDS“ bildet. 
betexten im eigentlichen Wortartikel. Das wiederum hat eine auf den ersten Blick nicht zu durchschauende Diskrepanz zwischen Datenmaterial und Wortartikel zu Folge.

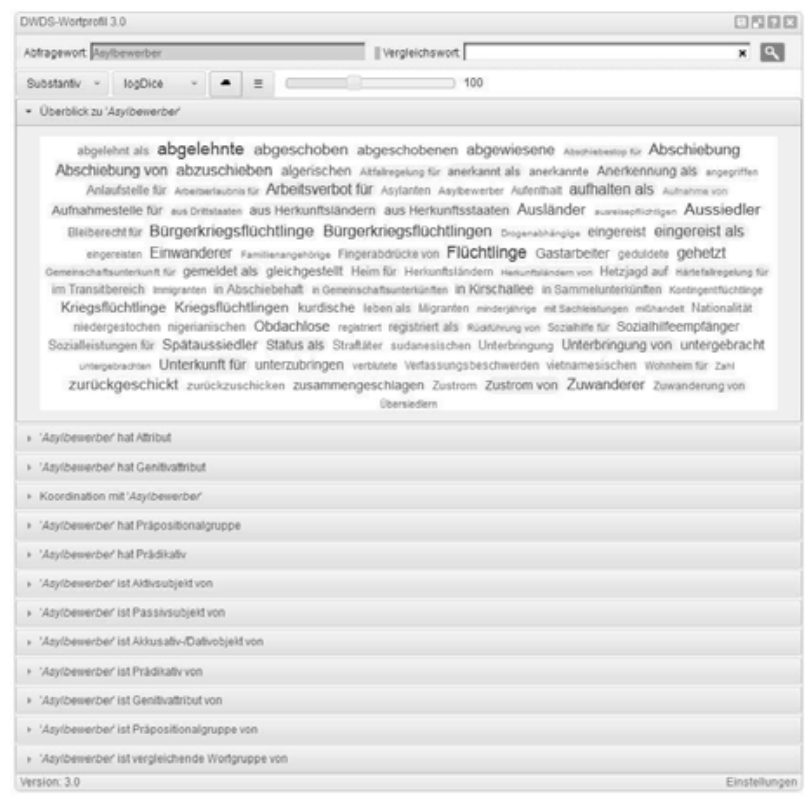

Abb. 2: Eintrag Asylbewerber, Ausschnitt (Panel) DWDS-Wortprofil 3.0 im „DWDS“

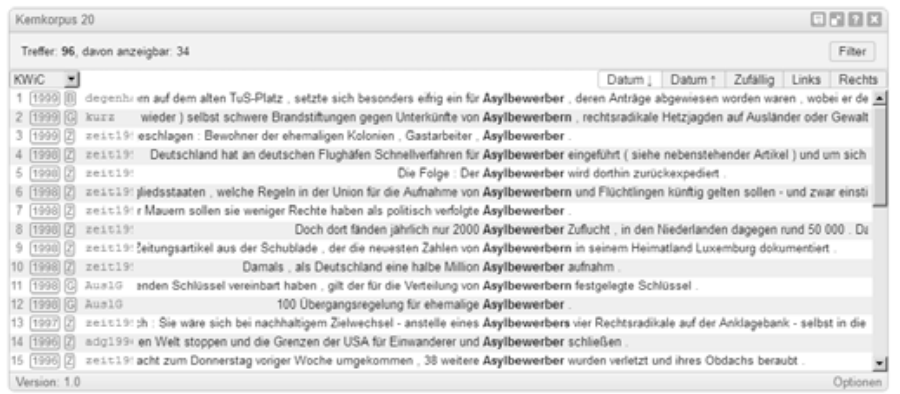

Abb. 3: Eintrag Asylbewerber, Ausschnitt (Panel) KWICs/Treffer im Kernkorpus im „DWDS“

Um die Problematik weiter einzukreisen, muss man einen ,Spezialisten ' bemühen, wie z.B. „Duden Richtiges und gutes Deutsch“. Doch dort sucht man Migrant, Flüchtling, Ausländer, Asylbewerber als Stichwörter zunächst vergeblich, bei Asylant stößt man immerhin auf folgenden Eintrag:

(6) Asylant: Diese Bezeichnung für »Asylbewerber« ist Ende der 70er-Jahre gebräuchlich geworden. Gelegentlich werden damit auch »Asylbewerber« und »Asylberechtigte« ohne die nötige Differenzierung gleichermaßen angesprochen. In jüngerer Zeit gibt es vereinzelt Kritik an der Verwendung des Wortes; man ist der Meinung, es sei zu einer Art Schimpfwort geworden, und möchte es deshalb im Sinne der 个Political Correctness nicht mehr gebrauchen.

In diesem Wortartikel wird das gesellschaftspolitische, rechtliche und moralische Potenzial bzw. die Brisanz dieser Begrifflichkeiten explizit formuliert, die Interpretation der Daten wird nicht den Nutzer/innen überlassen. Wesentlich deutlicher und umfassender wird der fünfseitige (!) Eintrag im Nachschlagewerk „Brisante Wörter“ (Strauß/Haß/ Harras 1989), dort heißt es u.a.: 
Asylant ist ein [...] aus Asyl und -ant geprägtes Wort. Dabei soll die Endung auf -ant vermutlich in vielfältigen Assoziationen vom Bummelanten bis zum Querulanten auf den ,schlechten' Charakter der so bezeichneten Person hinweisen. Denn viele ant-Substantive [...] werden häufig abwertend, manchmal sogar als Schimpfwörter verwendet. Mit dem Ausdruck Asylant werden Personen charakterisiert, die aus politischen, rassischen oder religiösen Gründen ihr Heimatland verlassen (müssen), weil sie, nach Einschätzung des Sprechers oft nur vorgeblich, dort politisch verfolgt, unterdrückt oder gefoltert werden und in einem anderen Land um Asyl bitten, sich um das Asylrecht bewerben, d.h. in dem betreffenden Land um Zuflucht, Schutz und unbefristeten Aufenthalt nachsuchen. (Strauß/Haß/Harras 1989, S. 86f.)

Die beschriebenen diskursiven Eigenheiten werden in speziellen sprachreflexiv ausgerichteten Wörterbüchern also sehr ausführlich erfasst. Als Lexikograf/in im Arbeitsbereich ,allgemeine Lexikografie' hingegen sieht man sich einer wahren Flut an Sprachdaten gegenüber, die man nicht alle zu Wörterbuchinformationen verdichten kann, deren adäquate Verarbeitung jedoch zumindest in den als signifikant einzustufenden Fällen kommentiert werden sollte. In vielen Fällen läuft es allerdings darauf hinaus, speziell die Daten zur Charakterisierung der ,Gebrauchsbesonderheiten' zu den hinlänglich bekannten wörterbuchpragmatischen Etikettierungen $\mathrm{zu}$ kondensieren, also etwa abwertend, veraltet usw. In traditionellen Printwörterbüchern kann dieses Vorgehen gewiss mit der Druckraumknappheit begründet werden. In der modernen allgemeinsprachigen Onlinelexikografie zählt dieses Argument allerdings nicht mehr, und es sollten andere Lösungen gefunden werden (vgl. Schnörch 2008).

Es scheint also erstrebenswert - so ließe sich resümieren -, dass sich die allgemeine Lexikografie irgendwo zwischen der traditionell allzu knappen bzw. verknappenden Herangehensweise und der sprachreflexiv bzw. diskurslexikografisch episch-narrativen positioniert: Es sollte darum gehen, Wörter mit kommunikativen bzw. diskursiven Besonderheiten des Gebrauchs neutral zu beschreiben, ohne dabei zu neutralisieren. Es wäre anzustreben, den ,problematischen' (insbesondere diskriminierenden, stigmatisierenden, euphemistischen usw.), also wertenden Sprachgebrauch zumindest kommentierend zu beschreiben (s.u. Abschnitt 3).

Ein anderer wesentlicher Faktor bei der Beschreibung kommunikativ bzw. diskursiv schwieriger Wörter ist der starke Zeitbezug ihrer Verwendung: Die Begriffe (und somit deren Bedeutung bzw. Bedeutungswandel) sind i.d.R. eng an zeitlich zu verortende Diskurse gebunden. Wandeln sich die Diskurse, wandelt sich entsprechend der Gebrauch der Wörter - zumindest ,nur' in Nuancen. Dies zeitnah zu dokumentieren war in gedruckten Wörterbüchern schlicht unmöglich. Im elektronischen Medium hingegen ist es theoretisch möglich, Diskursveränderungen zeitnah und äußerst schnell zu erfassen und zu dokumentieren. Die Voraussetzungen hierfür sind: permanente Korpusaktualisierungen, Anwendung von Sortieroptionen von Treffern nach Zeitspannen sowie unmittelbare Onlinepublikation - das praktische Problem ist, die erforderliche Zeit und das Personal hierfür aufzubringen; trotzdem haben die Onlinepublikationen neue Möglichkeiten der Aktualisierung von Einträgen eröffnet. Eine entscheidende Rolle dabei spielt auch die Rückkopplung der lexikografischen Arbeit an elektronische Korpora. Wortartikel werden nicht nur korpusgestützt erarbeitet, es ist auch möglich, die Korpora zeitnah zu aktualisieren und sie selbst in den Beschreibungsrahmen einzubeziehen, denn naturgemäß erfahren Wörter wie Asylant, Asylbewerber, Asylberechtigter auch Thematisierungen in metasprachlichen Diskussionen in der Sprachgemeinschaft: „Was eine lexikalische Einheit bedeutet, ist immer das Ergebnis einer Aushandlung zwischen den Mitgliedern einer Diskursgemeinschaft. Wie in jeder anderen Gemeinschaft lässt sich Einigkeit nicht voraussetzen“ (Teubert 2006, 
S. 57). Zahlreiche Belege im elexiko-Korpus (vgl. www.owid.de/wb/elexiko/glossar/elexiko-Korpus.html) belegen den ,Aushandlungsprozess'; die Begriffs- bzw. Metadiskussion wird auch durch Schreibung der Wörter in Anführungszeichen verdeutlicht.

(7) „Wir wollen so die Gemeinschaft ausländischer und einheimischer junger Menschen fördern", formulierte der Kreisbeigeordnete Hans-Josef Koggel das integrative Ziel der Wochen. „Brandstifter gibt es genug, wir müssen Jugendliche begeistern, aufeinander zuzugehen. " $\mathrm{Da}$ dies eine drängende Herausforderung ist, betonte Ruth Fischer vom Jugendgemeinschaftswerk der Caritas: „Soziale Armut und Sozialneid nehmen zu, Integrationshilfen, etwa Sprachkurse, werden zurückgefahren." Integration werde so immer schwerer, deshalb verstehe sich die Caritas als Lobby und Anwalt der Migranten. Ruth Fischer verwies auf die oft verzerrte Darstellung des Themas in den Medien. Undifferenziert werde etwa der negative besetzte Begriff „Asylant“ gebraucht, selten sei von „Asylbewerbern“ und „Asylberechtigten“ die Rede. So würden Vorurteile geschürt, die während der Interkulturellen Wochen vor allem durch Informationen zu anderen Kulturkreisen und gemeinsame Aktionen abgebaut werden sollen. (Rhein-Zeitung, 30.7.1999; Mehr Information soll die Toleranz fördern)

In diesem Beleg werden nicht nur die semantischen Eigenschaften und kommunikativen Besonderheiten des Wortes Migrant illustriert, sondern auch dessen Vernetzung mit Vokabeln aus dem begrifflichen Umfeld angedeutet: Asylant, Asylbewerber, Asylberechtigter.

Die Ausführungen und Stichproben deuten an, dass die Beschreibung kommunikativ bzw. diskursiv brisanter Wörter eine Herausforderung für die allgemeine Lexikografie darstellt, der sie jedoch gewachsen ist: Aus lexikografischer Sicht erweist sich weniger die Beschreibung des denotativen Kerns, sondern gerade des konnotativen/diskursiven Gehalts als entscheidend, um (neben dem bloßen, Verständnis ${ }^{6}$ ) die ,politisch korrekte' Verwendung für Nachschlagende zu dokumentieren und zu kommentieren. Speziell für diesen Bereich besitzt die moderne, allgemeinsprachige Lexikografie beste Voraussetzungen mit den Möglichkeiten der korpusgestützten Sprachdatenanalyse und zeitnahen Onlinepublikation. Schwieriger scheint es im redaktionellen lexikografischen Prozess, den auf diesem Gebiet häufig sehr schmalen Grat zwischen Deskriptivität und Präskriptivität, zwischen Musterhaftem und Einzelphänomen und auch zwischen Gegenwart und Sprachwandel zu meistern.

\section{Lexikografische Lösungsansätze}

Im allgemeinen, einsprachigen elektronischen Wörterbuch elexiko wurde versucht, einige Desiderata bei der Beschreibung brisanter Begriffe einzulösen. Den konzeptionellen Rahmen hierfür stellt ein Angabetyp ,Besonderheiten des Gebrauchs' dar, der u.a. Raum für die Analyse und (sprachreflexive) Beschreibung diskursiver bzw. kommunikativer Eigenheiten gibt (vgl. Schnörch 2008). Dafür gelten Grundsätze, die sich wie folgt benennen und skizzieren ließen:

Korpusrückkopplung: Grundlage der lexikografischen Beschreibung kontroverser Begriffe sind (elektronische) Korpora (das sog. elexiko-Korpus, vgl. www.owid.de/wb/ elexiko/glossar/elexiko-Korpus.html) und in Konsequenz eine am Sprachgebrauch orientierte Bedeutungsauffassung. Speziell im Bereich sprachreflexiver Analysen genügt es auch nicht, Informationen rein automatisch zu generieren - dieser Schritt, eine Kookkurrenzanalyse und -interpretation, ist vielmehr das Fundament für die redaktionelle Arbeit, die bei aller sprachreflexiver Motivierung stets deskriptiv ausgerichtet bleiben sollte. 
Aktualität: Die Beschreibung erfolgt auf der Basis möglichst aktueller Korpora, die im Idealfall ein periodisches Update erfahren. Die mikrodiachrone Komponente nuancierten Bedeutungswandels kann ggf. berücksichtigt werden: Feine diskursive Verwendungs- und mithin (konnotative) Bedeutungsänderungen von Wörtern, die sich in kurzen Zeiträumen vollziehen, lassen sich so dokumentieren.

Sachinformationen: Die Aufklärung über brisante Wörter erfordert i.d.R. auch die Vermittlung von Sachinformationen, was u.a. dadurch begründet ist, dass die zeithistorischen Umstände bzw. bestimmte kulturelle Kontexte der gegenwärtigen Sprachgemeinschaft nicht mehr präsent sind, dass folglich bestimmte verständniserhellende Umstände und Fakten des Diskurses beigesteuert werden müssen.

Ausführlichkeit: Die Beschreibung diskursiver Eigenheiten erfolgt nicht auf dem Wege bloßer Etikettierung, ihr wird mehr Raum gegeben, um sie angemessener zu gestalten. In elexiko geschieht das durch ausführliche Kommentare und Belege. Tendenziell bewegen sich die Lexikograf/innen also in Richtung Diskurswörterbuch, ohne dessen Ansprüche und Ausführlichkeit erreichen zu können (und zu wollen).

Zugriffsmöglichkeiten: Wörter mit diskursiven (Bedeutungs-)Eigenheiten entfalten diese vornehmlich in bestimmten Diskursen. Konsequenterweise lassen sich die betreffenden Wörter zusammenfassen, gruppieren. Das Offenlegen derartig vernetzter Strukturen kann dazu beitragen, die lexikografische Analyse und Beschreibung adäquater zu gestalten, wie am Beispiel Asylant, Asylbewerber, Asylberechtigter schon angedeutet (s.o.). Besonders offenkundig werden wortübergreifende Zusammenhänge bei der sog. Euphemismustretmühle: Werden negative Verhältnisse, Einstellungen usw. nicht geändert, wird auch jede beschönigende Bezeichnung dafür im Laufe der Zeit wieder negativ konnotiert, bis diese erneut ersetzt wird, im Laufe der Zeit negativ konnotiert wird usw. In diesem Kontext lassen sich auch Ausländer, Gastarbeiter, Migrant, Person mit Migrationshintergrund (möglicherweise) aufschlussreicher betrachten, analysieren und beschreiben.

So entstehen in der Wirklichkeit sprachliche Vernetzungsstrukturen, die sich idealerweise auch im Wörterbuch widerspiegeln, etwa in Form von Wortartikeln, die konzeptionell nicht nur Informationen zu einem Wort, sondern einer ganzen Wortgruppe zusammenfassen sowie vernetzend und vergleichend präsentieren. Für die allgemeine einsprachige Lexikografie wäre ein derartiger Artikeltyp u.U. eine Möglichkeit, diskursrelevante Wörter in einem sprachreflexiven Rahmen adäquat, zusammenhängend und innovativ zu beschreiben.

In elexiko haben die Lexikograf/innen die vier ersten Punkte weitgehend umgesetzt, was nachfolgendes Beispiel illustrieren soll: ${ }^{3}$ Als allgemeinsprachiges Wörterbuch bietet elexiko korpusgestützt erarbeitete Informationen zu folgenden lesartenbezogenen Angabetypen: Bedeutungserklärung (u.a. Diskursangaben), Kollokationen, Konstruktionen, Sinnverwandte Wörter, Grammatik und Gebrauchsbesonderheiten (sowie für das Verständnis grundlegende Sachinformationen). Für das Stichwort Asylbewerber finden Nutzer/innen unter der Lesart ,Aufenthaltsrecht beantragende Person“ folgende Details:

Eine kleinere Anzahl von sog. Wortgruppenartikeln kann ab Frühjahr 2015 unter www.elexiko.de aufgerufen werden, beispielsweise auch zu den diskursrelevanten Personenbezeichnungen, die im vorliegenden Aufsatz diskutiert werden. 


\section{Sachinformationen ${ }^{4}$}

Sachinformationen zu Asylbewerber und zur Regelung des Asylrechts im Grundgesetz finden sich beispielsweise unter $<$ wissen-digital.de $>$ (zuletzt eingesehen am 6.2.2013). Weiteres Sachwissen zur Asylsuche findet sich außerdem unter $<$ Planet Wissen $>$ (zuletzt eingesehen am 5.3.2013).

\section{Einstellung des Sprechers}

Bei Asylbewerber handelt es sich um ein im nationalen wie im internationalen (Menschen-)Recht mitunter schwierig zu klärenden Begriff, der im elexiko-Korpus entsprechend kontrovers und emotional diskutiert wird (vgl. den Beleg). $\mathrm{Zu}$ der rein rechtlichen Seite gesellt sich eine humanitäre und moralische Dimension, welche die Komplexität des Begriffs noch verstärkt und zugleich eine höchst emotionale Ebene mit ins Spiel bringt. Asylbewerber wird im elexiko-Korpus häufig in Kontexten verwendet, in denen deren Status thematisiert, häufig eine pauschalisierte, vorurteilsgeprägte Meinung über Asylbewerber in Deutschland zum Ausdruck gebracht wird: Asylbewerbern werden in diesem Zusammenhang mitunter unlautere Beweggründe für eine Einreise unterstellt, z.B. rein wirtschaftliche Motivation bzw. kriminelle Energien (vgl. die Belege). In diesem Zusammenhang geht es im elexiko-Korpus oft um die Einstellung der Deutschen gegenüber Asylbewerbern. Diese ist zwischen den Polen Verständnis, Gastfreundschaft, Mitleid und Misstrauen, Vorurteil, Angst, rechter Propaganda angesiedelt. Im Dunstkreis von Schlagwörtern wie Ausländerfeindlichkeit, Kriminalisierung bzw. Kriminalität, nationale Gesinnung, Furcht vor dem Fremden und vor Ausnutzung prallen in diesem Spannungsfeld häufig mehr vom Gefühl als vom Verstand geleitete Positionen aufeinander, die sich im Extremfall auch in Straftaten entladen (vgl. die Belege). [...]

\section{Themengebundene Verwendung(en)}

Im Kontext von Politik, Recht und Wirtschaft

Im elexiko-Korpus wird Asylbewerber häufig im Spannungsfeld von Politik, Recht und Wirtschaft und somit als gesellschaftlich relevanter Begriff verwendet, z.B. wenn es um Fragen der Humanität, des Status, der Integration und allgemein um Aspekte einer offenen Gesellschaft im Kontext der sich zunehmend vernetzenden Welt geht (vgl. die Belege). [...]

Obwohl elexiko ein allgemeinsprachiges Wörterbuch ist, können auch in diesem Rahmen Informationen angeboten werden, die über die Angaben konventioneller Wörterbücher hinausgehen, und die einen wichtigen Beitrag zur Beschreibung des korrekten Gebrauchs sozio-kulturell oder politisch kontroverser Ausdrücke leisten. Diese Angaben erfüllen zumindest zweierlei Aufgaben: Sie sensibilisieren Nachschlagende, denen die Kontroversität nicht bewusst ist (z.B. Nichtmuttersprachler); sie helfen (mit Blick auf die Sprachproduktion), bei der Wortwahl Unsicherheiten in Bezug auf ggf. umstrittene Verwendungsweisen zu klären.

\section{4. $\quad$ Fazit und Ausblick}

In elexiko wird der, Wortschatz des öffentlichen Sprachgebrauchs' (als Modul) in den lexikografischen Beschreibungsmittelpunkt gerückt, was maßgeblich durch das zugrunde gelegte Zeitungskorpus bedingt ist. In diesem Rahmen spielen auch kommunikativ bzw. diskursiv ,brisante" Wörter eine auffällige Rolle, sodass auch die Beschreibung von Gebrauchsbesonderheiten bei den Einzelwortartikeln mehr Aufmerksamkeit zuteil wurde

\footnotetext{
4 Hier und im Folgenden handelt es sich um wörtliche Zitate aus elexiko.
} 
und wird. Dies geschieht bislang stets mit Fokus auf die Beschreibung des Einzelworts im Einzelwortartikel. Gerade dieser Wortschatzbereich bietet sich jedoch für eine, feldmäßige' Bearbeitung und auch Präsentation an: Auch Haß (2006) wies darauf hin, dass es durchaus sinnvoll sein kann, ,thematisch gebündelte Wortschatzbereiche auf der Basis möglichst aktueller Quellen zeitnah zu publizieren“ (ebd., S. 77). Recherchemöglichkeiten in elektronischen Wörterbüchern ermöglichen vielfach auch onomasiologische Zugriffsmöglichkeiten - allerdings i.d.R. auf eine Summe von Einzelwortartikel. Ein Schritt in die richtige Richtung wäre, die entsprechenden Artikel gebündelt durch den gleichen Lexikografen bearbeiten zu lassen, da auch ein solches Verfahren den kontextsensitiven Blick über den Einzelartikel hinaus ermöglicht. Im Idealfall ließen sich Bezeichnungen für Personen mit Migrationshintergrund von vornherein konzeptionell in Wortgruppenartikeln erfassen: Das ermöglicht sowohl die explizite Kontrastierung von Gemeinsamkeiten und Unterschieden, den ,kontextsensitiven' Blick auf Einzelbedeutungen als auch den Überblick über das begriffliche Umfeld (Ausländer, Gastarbeiter, Migrant, Asylant, Asylbewerber, Asylberechtigter usw.). Stets die entsprechende Aufbereitung der Daten vorausgesetzt, erhielten Nutzerinnen und Nutzer eines derartigen Wörterbuches u.a. Einblick in diskursive Zusammenhänge der Einzelwörter (Gemeinsamkeiten und Abgrenzungen) sowie mikrodiachrone Entwicklungen (z.B. im Zeitverlauf erfolgende Wortersetzungen bei der sog. Euphemismustretmühle). $\mathrm{Ob}$ in diesem Zusammenhang auch andere ,Felder" - etwa Gründe für Migration, z.B. Globalisierung, Krieg, Konflikt, Wirtschaftskrise in die Betrachtungen einzubeziehen sind, müsste geprüft werden. Theoretisch ließen sich so sprachliche Zusammenhänge zeigen, indem man von Feld zu Feld ,wandern' und thematische Brücken sichtbar machen könnte. Dadurch ließe sich ggf. auch sprachlicher Wandel aufdecken, da man sähe, wo sich welche thematischen Bezüge auftun.

Betrachtet und vergleicht man in elexiko diskursrelevante Personenbezeichnungen wie Ausländer (Lesart: ,Person aus einem fremden Land'), Flüchtling (,Migrant') und Asylbewerber (,Aufenthaltsrecht beantragende Person'), so wird schnell deutlich, dass nicht nur unter der Rubrik , Gebrauchsbesonderheiten' sprachreflexive Kommentare zu finden sind. Auch eine Kontrastierung von Kollokatoren in der Rubrik ,Kollokationen“ hilft in der ,Wortgruppenperspektive' bei der richtigen sprachlichen Verortung des Stichworts in der jeweiligen Einzelbedeutung weiter. Besonders deutlich wird dies, wenn man jene Kollokatoren gegenüberstellt, die auf textliche Thematisierungen hinweisen (vgl. Tab. 1). 


\begin{tabular}{|c|c|c|}
\hline $\begin{array}{l}\text { Arbeitslosigkeit } \\
\text { Asyl } \\
\text { Asylpolitik } \\
\text { Aufenthalt } \\
\text { Aufenthaltserlaubnis } \\
\text { Aufenthaltsgenehmigung } \\
\text { Bleiberecht } \\
\text { Deutschkurse } \\
\text { Einbürgerungstest } \\
\text { Herkunftsland } \\
\text { Integrationskurse } \\
\text { Kriminalität } \\
\text { Menschenhandel } \\
\text { Nationalität } \\
\text { Parolen } \\
\text { Rechte } \\
\text { Scheinehe } \\
\text { Sprachkurse } \\
\text { Staatsbürgerschaft } \\
\text { Stimmrecht } \\
\text { Straftaten } \\
\text { Toleranz } \\
\text { Wahlrecht }\end{array}$ & $\begin{array}{l}\text { Was wird in Zusammenhang } \\
\text { mit einem Flüchtling } \\
\text { thematisiert? }\end{array}$ & $\begin{array}{l}\text { Was wird in } \\
\text { Zusammenhang mit } \\
\text { Asylbewerber thematisiert? } \\
\text { Abschiebehaft } \\
\text { Abschiebestopp } \\
\text { Altfallregelung } \\
\text { Arbeitserlaubnis } \\
\text { Arbeitsverbot } \\
\text { Ausländerbehörde } \\
\text { Ausschreitungen } \\
\text { Bleiberecht } \\
\text { Brandanschlag } \\
\text { Chipkarte } \\
\text { Erstaufnahme } \\
\text { Heimatland } \\
\text { Heiratsverbot } \\
\text { Hetzjagd } \\
\text { Hungerstreik } \\
\text { Residenzpflicht } \\
\text { Sozialhilfe } \\
\text { Überfall } \\
\text { Übergriff } \\
\text { Unterbringungskosten } \\
\text { Verfassungsbeschwerde } \\
\text { Kommentar: } \\
\text { Einige Mitspieler wie } \\
\text { Brandanschlag, Überfall } \\
\text { und Hetzjagd zeugen von } \\
\text { Berichterstattungen über } \\
\text { Gewalt- und Straftaten } \\
\text { mit ausländerfeindlichem } \\
\text { Hintergrund in den Texten } \\
\text { des elexiko-Korpus. }\end{array}$ \\
\hline
\end{tabular}

Tab. 1: Vergleichende Gegenüberstellung der ,thematischen Kollokatoren“ von Ausländer, Flüchtling und Asylbewerber

Die skizzierten Beispiele zeigen, dass in elexiko eine sehr differenzierte Beschreibung sprachlicher, diskursrelevanter Eigenschaften von Einzelwörtern (in bestimmten Lesarten) vorgenommen wird - differenzierter und kohärenter als in vielen anderen Wörterbüchern. Dafür sorgen nicht nur die entsprechende Formulierung der semantischen Paraphrase sowie die Belegbeispiele aus dem elexiko-Korpus und ausführliche Kommentare (und Belege) im Informationstyp ,Gebrauchsbesonderheiten'; auch die anderen Angabetypen lassen Rückschlüsse auf diskursive Besonderheiten zu, etwa die Informationen unter der Rubrik ,Kollokationen'. Hier wiederum ist es insbesondere der vergleichende Ansatz, der zugleich die Perspektive vom Einzelwortartikel zur kontrastierenden Perspektive von Wortgruppenartikeln verschiebt (vgl. den Beispielvergleich der Kollokatoren in 
Tab. 1). Wie schon bei den Einzelwortartikeln sollte gerade bei derartigen Wortgruppenartikeln Qualität und Zuverlässigkeit der Daten und Informationen gewährleistet werden. In elexiko geschieht dies mittels einer gut abgestimmten Verzahnung korpuslinguistischer Methoden und Verfahren mit redaktionellen Arbeitsschritten (zum lexikalischen Prozess vgl. z.B. Schnörch 2005).

Verlegt man den Fokus der lexikografischen Beschreibung jedoch vom Einzelwort auf Wortgruppen, müssen möglicherweise auch diese Arbeitsschritte überdacht und modifiziert werden, da die gestiegene Komplexität von ,Vernetzungsartikeln' den redaktionell zu bewältigenden Arbeitsanteil erhöht: Die Menge der Daten steigt beim vergleichenden Ansatz mit der Zahl der in den Vergleich einzubeziehenden Wörter - man denke nur an das manuelle bzw. visuelle Kontrastieren von zwei, drei und mehr Kollokationsprofilen.

Möglicherweise können hier weitere (und ergänzende) Verfahren und Methoden der Korpuslinguistik erprobt und eingesetzt werden, die beispielsweise den Vernetzungsaspekt stärker berücksichtigen. Auf einen solchen Ansatz soll ausblickend noch verwiesen werden. Dessen lexikografischer Einsatz wurde im Hinblick auf Synonyme (vgl. Marková 2012) und sog. Paronyme (z.B. effektiv vs. effizient, vgl. Storjohann 2013) bereits erprobt bzw. untersucht.

Für die vergleichende Analyse von Paronymen hat Petra Storjohann topographische Profile, sog. SOMs (Self-Organising Feature Maps, vgl. Kohonen 1984, 1990; Keibel/Belica 2007; Vachková/Belica 2009) getestet und dokumentiert:

SOMs positionieren lexikalische Ausdrücke und lexikalische Einheiten in einer zweidimensionalen, gitter- bzw. netzartigen Struktur auf die Weise, dass jeweils benachbarte Matrixzellen/Gitterkästchen semantische Nähe zwischen Kollokationsprofilen der darin aufgeführten Ausdrücke implizieren. (Storjohann 2013, S. 406)

Die für Gastarbeiter mittels SOM erstellte Grafik zeigt Abbildung 4.

Derartige Methoden und Verfahren erlauben es, Sprachdaten nicht nur auf einer zweidimensionalen, horizontalen (kollokativen) Ebene vorzustrukturieren, sondern gewissermaßen auch die vertikale Ebene miteinzubeziehen: Wörter werden in grau (eigentlich: farblich) abgestuften Gruppen zusammengefasst, die je nach Farbabstufung graduell (sehr) ähnliche bis (sehr) verschiedene Kollokationsprofile aufweisen.

Die differenzierte, zuverlässige, qualitativ anspruchsvolle lexikografische Beschreibung von Wörtern oder Wortgruppen setzt korpusgestützte Verfahren voraus, um die Datenmenge überhaupt verarbeiten zu können. Besonders für den Bereich der allgemeinen, auch sprachreflexiven bzw. auf den Diskurs ausgerichteten Lexikografie genügen vollautomatisierte Methoden nicht, um dem Anspruch von Qualität und Zuverlässigkeit und Verstehbarkeit gerecht zu werden. 


\begin{tabular}{|c|c|c|c|c|}
\hline $\begin{array}{l}\text { Nordafrika } \\
\text { Maghreb } \\
\text { nordafrikanisch } \\
\text { Marokko } \\
\text { Tunesien } \\
\text { Urlauber } \\
\text { Ågypten } \\
\text { Indonesien }\end{array}$ & $\begin{array}{l}\text { schwarzafrikanisch } \\
\text { Nachbarland } \\
\text { Türkei } \\
\text { Anrainerstaat } \\
\text { Kleinasien } \\
\text { Algerien } \\
\text { Westsahara } \\
\text { weiterreisen }\end{array}$ & $\begin{array}{l}\text { Kriegsgebiet } \\
\text { Kroatien } \\
\text { Mazedonien } \\
\text { Albanien } \\
\text { Glaubensbruder } \\
\text { geflohen } \\
\text { Exil } \\
\text { Anatolien }\end{array}$ & $\begin{array}{l}\text { serbisch } \\
\text { makedonisch } \\
\text { Adriaküste } \\
\text { Greueltat } \\
\text { Kampfgebiet } \\
\text { Kriegsverbrechen } \\
\text { Kriegsopfer } \\
\text { desertiert }\end{array}$ & $\begin{array}{l}\text { Söldner } \\
\text { Truppe } \\
\text { Soldat } \\
\text { Nationalist } \\
\text { Sicherheitskraft } \\
\text { Armee } \\
\text { Sicherheitskräfte } \\
\text { Delegation }\end{array}$ \\
\hline $\begin{array}{l}\text { eingewandert } \\
\text { einwandern } \\
\text { Zielland } \\
\text { Transitland } \\
\text { Bootsflüchtling } \\
\text { osteuropäisch } \\
\text { Deutschland } \\
\text { Osteuropa }\end{array}$ & $\begin{array}{l}\text { Rumänien } \\
\text { Landweg } \\
\text { Ostblockstaat } \\
\text { Nachfolgestaat } \\
\text { Apulien } \\
\text { aus } \\
\text { zurückgekehrt } \\
\text { Pole }\end{array}$ & $\begin{array}{l}\text { Zuflucht } \\
\text { geflüchtet }\end{array}$ & $\begin{array}{l}\text { Vertriebene } \\
\text { Albaner } \\
\text { Serbe } \\
\text { Volksgruppe } \\
\text { moslemisch } \\
\text { Nordteil } \\
\text { Moslem } \\
\text { Kosovo }\end{array}$ & $\begin{array}{l}\text { Bevölkerungsmehrheit } \\
\text { Enklave } \\
\text { Extremist }\end{array}$ \\
\hline $\begin{array}{l}\text { eingereist } \\
\text { Einreise } \\
\text { ausreisen } \\
\text { Herkunftsland } \\
\text { Schlepperbande } \\
\text { einreisen } \\
\text { Heimatland } \\
\text { zurückgeschickt }\end{array}$ & $\begin{array}{l}\text { Kriegsflüchtling } \\
\text { abgeschoben } \\
\text { Wirtschaftsflüchtling } \\
\text { Rückführung } \\
\text { abschieben } \\
\text { Auffanglager } \\
\text { Visumpflicht } \\
\text { zurückschicken }\end{array}$ & $\begin{array}{l}\text { Flüchtling } \\
\text { Staatsangehörige } \\
\text { Flüchtlingsfamilie } \\
\text { lebend } \\
\text { Flüchtlingskind } \\
\text { Asiat } \\
\text { Asiate } \\
\text { Vietnamese }\end{array}$ & $\begin{array}{l}\text { Türke } \\
\text { Kurde } \\
\text { Kambodschaner } \\
\text { Tamile } \\
\text { Bosnier } \\
\text { bewohnt } \\
\text { bewohnen } \\
\text { Volkszugehörigkeit }\end{array}$ & $\begin{array}{l}\text { muslimisch } \\
\text { Minderheit } \\
\text { Muslime } \\
\text { Diaspora } \\
\text { Bevölkerungsanteil } \\
\text { Bevölkerungsgruppe } \\
\text { Bevölkerungsteil } \\
\text { drangsaliert }\end{array}$ \\
\hline $\begin{array}{l}\text { illegal } \\
\text { Drittstaat } \\
\text { Einwanderungsland } \\
\text { Ausschaffung } \\
\text { Immigration } \\
\text { ausreisepflichtig } \\
\text { Visum }\end{array}$ & $\begin{array}{l}\text { Bürgerkriegsflüchtling } \\
\text { Asylant } \\
\text { Asylbewerber } \\
\text { Vertragsarbeiter } \\
\text { Abschiebung } \\
\text { Asylsuchende } \\
\text { straffällig } \\
\text { Asylwerber }\end{array}$ & $\begin{array}{l}\text { Immigrant } \\
\text { Einwanderer } \\
\text { deutschstämmig } \\
\text { Immigrantin } \\
\text { staatenlos } \\
\text { Heimatvertriebene } \\
\text { Umsiedler }\end{array}$ & $\begin{array}{l}\text { zugewandert } \\
\text { Emigrant } \\
\text { assimilieren } \\
\text { türkischstämmig } \\
\text { vorwiegend } \\
\text { assimiliert }\end{array}$ & $\begin{array}{l}\text { Staatsbürger } \\
\text { Abstammung } \\
\text { Mitbürger } \\
\text { Nationalität } \\
\text { Staatsbürgerin } \\
\text { Herkunft } \\
\text { Großfamilie } \\
\text { jüdisch }\end{array}$ \\
\hline $\begin{array}{l}\text { Staatsangehörigkeit } \\
\text { Arbeitserlaubnis } \\
\text { Aufenthaltsgenehmigun } \\
\text { Aufenthaltsstatus } \\
\text { Arbeitsgenehmigung } \\
\text { Familiennachzug } \\
\text { Familienzusammenführ } \\
\text { Arbeitsbewilligung }\end{array}$ & $\begin{array}{l}\text { Ausländer } \\
\text { Spätaussiedler } \\
\text { Zustrom } \\
\text { Integration } \\
\text { Zuzug } \\
\text { Kontingentflüchtling } \\
\text { Einwanderung } \\
\text { Deutschkurs }\end{array}$ & $\begin{array}{l}\text { Zuwanderer } \\
\text { Aussiedler } \\
\text { Saisonarbeiter } \\
\text { Arbeitskraft } \\
\text { Übersiedler } \\
\text { Neubürger } \\
\text { Schwarzarbeiter } \\
\text { Sozialhilfeempfänger }\end{array}$ & $\begin{array}{l}\text { Fremdarbeiter } \\
\text { Wanderarbeiter } \\
\text { Auswanderer } \\
\text { Mitbürgerin } \\
\text { zumeist } \\
\text { Erntehelfer }\end{array}$ & $\begin{array}{l}\text { Landarbeiter } \\
\text { Bauarbeiter } \\
\text { Tourist } \\
\text { Tagelöhner } \\
\text { Hausangestellte } \\
\text { Seeleute } \\
\text { Putzfrau } \\
\text { Nachfahr }\end{array}$ \\
\hline
\end{tabular}

Abb. 4: SOM für Gastarbeiter

SOMs (vgl. Abb. 4) bieten gewiss eine Fülle strukturierter Daten für die, die sie interpretieren können. Es darf jedoch gemutmaßt werden, dass potenzielle Nutzer/innen allgemeinsprachiger Wörterbücher i.d.R. mit der bloßen Präsentation von ,Rohdaten' wenig anfangen können. Daher sollte es im Interesse einer akademischen Lexikografie sein, diese Daten ,zu vertexten', sie methodisch auswertend, interpretierend, zuverlässig, kommentierend, usw. sowie erzählend für Nutzer/innen zu übersetzen, um auf diese Weise zugleich einen wissenschaftlichen, lexikografischen Mehrwert zu schaffen. Zu diesem Zweck bedarf es eines sinnvollen Miteinanders von Korpuslinguist/innen und Lexikograf/ innen, von automatisierten und redaktionell-interpretativen Arbeitsschritten.

Die diskurslexikografische bzw. sprachreflexive Beschreibung im Rahmen der allgemeinsprachigen Lexikografie könnte also von den gegenwärtigen Entwicklungen profitieren und sollte sie nutzen - geht es auf diesem Gebiet doch nicht ,nur' um quantitative Aspekte von 
punktueller Rechercheökonomisierung und Zugriffszahlenerhöhung; speziell dieser Sektor bietet sich an für die gehaltvolle Dokumentation von zeithistorischer, kontextbezogener sprachlicher Kultur (und Unkultur), für das Schaffen eines akademisch-lexikografischen, allgemeinsprachig formulierten Mehrwerts: Er ist Voraussetzung und Mittel, um zuverlässig und verständlich befriedigende Antworten auf sprachkritische Fragen geben zu können. Und genau das würden sowohl textproduzierende wie -rezipierende Interessenten gewiss zu schätzen wissen, ganz gleich, ob sie als Journalisten oder Lehrerinnen tätig sind.

\section{Literatur}

Duden (2001): Das Universalwörterbuch. Hrsg. vom Wissenschaftlichen Rat der Dudenredaktion: A. Klosa, K. Kunkel-Razum, W. Schulze-Stubenrecht, M. Wermke. 4. völlig neu bearb. und erw. Auflage. Mannheim u.a.: Dudenverlag.

Duden (2007): Richtiges und gutes Deutsch. Hrsg. von der Dudenredaktion. 6. vollständig überarbeitete Auflage. Mannheim u.a: Dudenverlag. (= Duden 9).

Duden (2010): Das Bedeutungswörterbuch. Hrsg. von der Dudenredaktion. 4. neu bearbeitete und erweiterte Aufl. Mannheim u.a.: Dudenverlag. (= Duden 10).

Elsner-Petri, Sabine (in Druck): Political Correctness (PC) in gegenwartssprachlichen einsprachigen Bedeutungswörterbüchern. In: Kilian, Jörg/Eckhoff, Jan (Hg.): Deutscher Wortschatz - beschreiben, lernen, lehren. Beiträge zur Wortschatzarbeit in Wissenschaft, Sprachunterricht, Gesellschaft. Frankfurt u.a. (= Germanistik Didaktik - Unterrich 12).

Haß, Ulrike (2006): Wörterbücher und Wissen über Wörter, auch eine Kulturgeschichte. In: Der Deutschunterricht 1/2006, S. 69-78.

Jung, Matthias/Niehr, Thomas/Böke, Karin (Hg.) (1997): Die Sprache des Migrationsdiskurses. Wiesbaden: Westdeutscher Verlag.

Jung, Matthias/Niehr, Thomas/Böke, Karin (2000): Ausländer und Migranten im Spiegel der Presse. Wiesbaden: Westdeutscher Verlag.

Kämper, Heidrun (2013): Wörterbuch zum Demokratiediskurs 1967/68. Unter Mitwirkung von Elisabeth Link. Berlin: Akademie-Verlag. Online unter: http://intern.owid.de/wb/disk68/start.html.

Keibel, Holger/Cyril Belica (2007): CCDB. A Corpus-Linguistic Research and Development Workbench. Proceedings of the 4th Corpus Linguistics Conference (CL 2007), Birmingham. Online unter: www. birmingham.ac.uk/documents/college-artslaw/corpus/conference-archives/2007/134Paper.pdf.

Kohonen, Teuvo (1984): Self-Organization and Associative Memory. Berlin: Springer.

Kohonen, Teuvo (1990): The Self-Organizing Map. New Concepts in Computer Science. Proceedings of Symposium in Honor of Jean-Claude Simon, Paris. S. 181-190.

Marková, Vera (2012). Synonyme unter dem Mikroskop. Eine korpuslinguistische Studie. Tübingen: Narr.

Niehr, Thomas/Böke, Karin (Hg.) (2000): Einwanderungsdiskurse. Wiesbaden: Westdeutscher Verlag.

Schnörch, Ulrich (2005): Der Autoren-Arbeitsplatz: Ein elexiko-Wortartikel entsteht. In: Haß, Ulrike (Hg.) (2005): Grundfragen der elektronischen Lexikographie. elexiko - das Online-Informationssystem zum deutschen Wortschatz. Berlin/New York: de Gruyter. S. 105-130. (= Schriften des Instituts für Deutsche Sprache 12).

Schnörch, Ulrich (2008): Von der Markierung zur Beschreibung: Besonderheiten des (Wort-)Gebrauchs in elexiko. In: Bernal, Elisenda/Cesaris, Janet (Hg.): Proceedings of the Thirteenth EURALEX International Congress, Barcelona, Spain, July 15th-19th, 2008. (Sèrie Activitats 20). Barcelona: Institute Universitari de Linguistica Aplicada. S. 607-616.

Storjohann, Petra (2013): Korpuslinguistische und lexikografische Ansätze zur Beschreibung deutscher Paronyme. In: Sava, Doris/Schuringer, Hermann (Hg.). Im Dienste des Wortes. Lexikologische und lexiko- 
grafische Streifzüge; Festschrift für Ioan Lazarescu. Passau: Stutz. S. 401-418. (= Forschungen zur deutschen Sprache in Mittel-, Ost- und Südosteuropa / FzDiMOS 3).

Stötzel, Georg/Eitz, Thorsten (Hg.) (2002): Zeitgeschichtliches Wörterbuch der deutschen Gegenwartssprache. Hildesheim u.a.: Olms.

Strauß, Gerhard/Haß, Ulrike/Harras, Gisela (1989): Brisante Wörter von Agitation bis Zeitgeist. Berlin/New York: de Gruyter. (= Schriften des Instituts für deutsche Sprache 29.

Teubert, Wolfgang (2006): Korpuslinguistik, Hermeneutik und die soziale Konstruktion der Wirklichkeit. In: Linguistik online 28/3, 2006, S. 41-60. Online unter: https://bop.unibe.ch/linguistik-online/issue/view/162.

Vachková, Marie/Cyril Belica (2009): Self-Organizing Lexical Feature Maps. Semiotic Interpretation and Possible Application in Lexicography. Interdisciplinary Journal for Germanic Linguistics and Semiotic Analysis 13/2, S. 223-260.

Wengeler, Martin (1995): Multikulturelle Gesellschaft oder Ausländer raus? Der sprachliche Umgang mit der Einwanderung seit 1945. In: Stötzel, Georg et al. (1994): Kontroverse Begriffe. Geschichte des öffentlichen Sprachgebrauchs in der Bundesrepublik Deutschland. Berlin/New York: de Gruyter. S. 711-749.

Wengeler, Martin (2010): Entwicklungsländer, Gastarbeiter, Schwangerschaftsunterbrechung. Formen und Funktionen semantischer Remotivierungen im öffentlich-politischen Sprachgebrauch. In: Harnisch, Rüdiger (Hg.): Prozesse sprachlicher Verstärkung. Berlin/New York: de Gruyter. S. 213-227.

\section{Elektronische Ressourcen (Stand: 31.7.2014)}

AntiDiskriminierungsBüro (ADB) Köln/Öffentlichkeit gegen Gewalt e.V. (Hg.) (2013): Leitfaden für einen rassismuskritischen Sprachgebrauch. Handreichung für Jorunalist_innen. Online unter: www.adbsachsen.de/tl_files/adb/pdf/Leitfaden_ADB_Koeln_disfreie_Sprache.pdf.

Belica, Cyril (1995): Statistische Kollokationsanalyse und -clustering. Korpuslinguistische Analysemethode. Institut für Deutsche Sprache, Mannheim.

Belica, Cyril (2001ff.): Kookkurrenzdatenbank CCDB - V3.3. Eine korpuslinguistische Denk- und Experimentierplattform. Mannheim. Online unter: http://corpora.ids-mannheim.de/ccdb/.

Diskurswörterbuch zum ,Protestdiskurs 67/68‘. Online unter: www.owid.de/wb/disk68/start.html

DWDS. Digitales Wörterbuch der Deutschen Sprache. www.dwds.de/

Duden online: www.duden.de/woerterbuch

elexiko: www.owid.de.

elexiko-Korpus: www.owid.de/wb/elexiko/glossar/elexiko-Korpus.html

PONS-Wörterbuch Deutsch als Fremdsprache (online): www.pons.com

Dr. Ulrich Schnörch

Institut für Deutsche Sprache

R 5, 6-13

D-68161 Mannheim

E-Mail: schnoerch@ids-mannheim.de 